
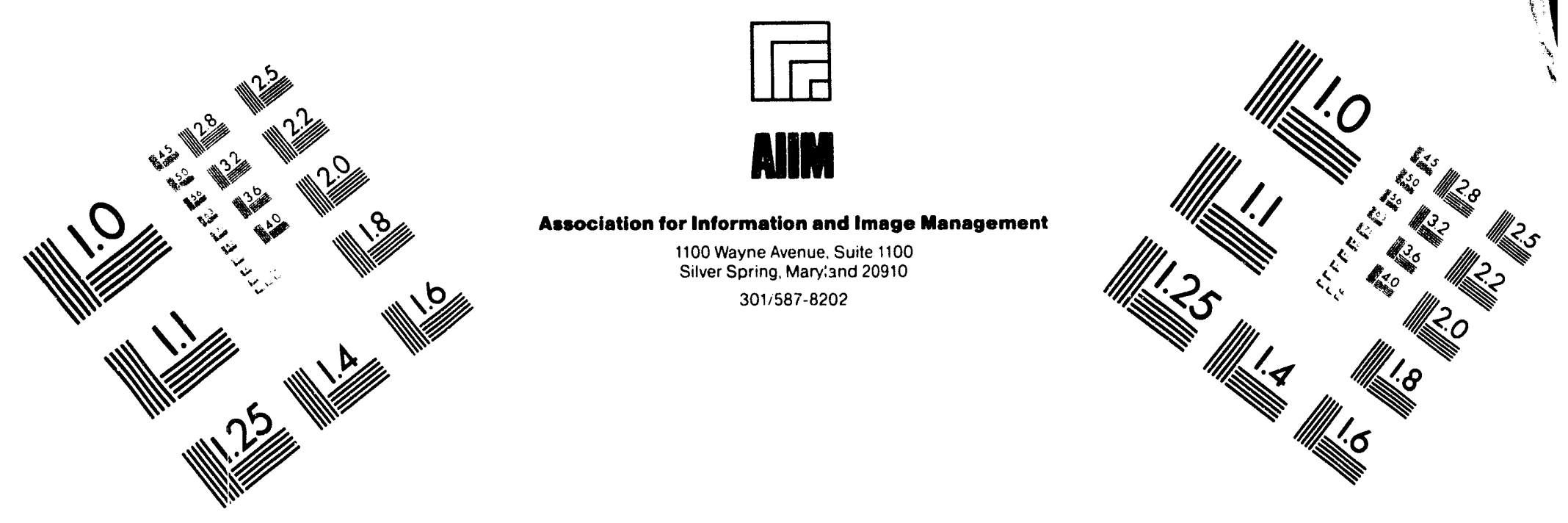

Centimeter

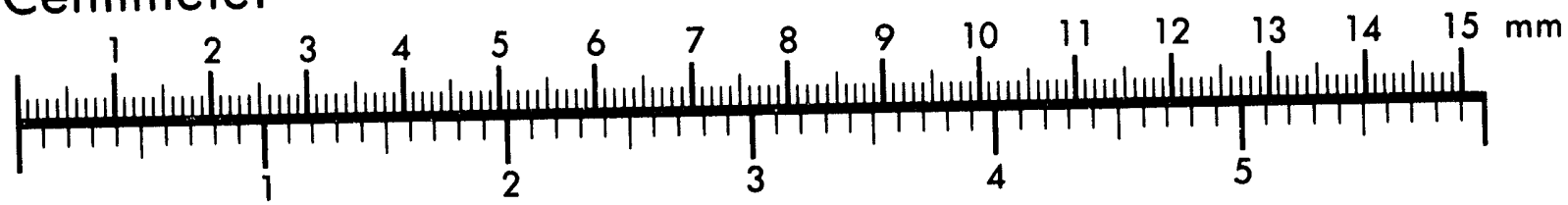

Inches
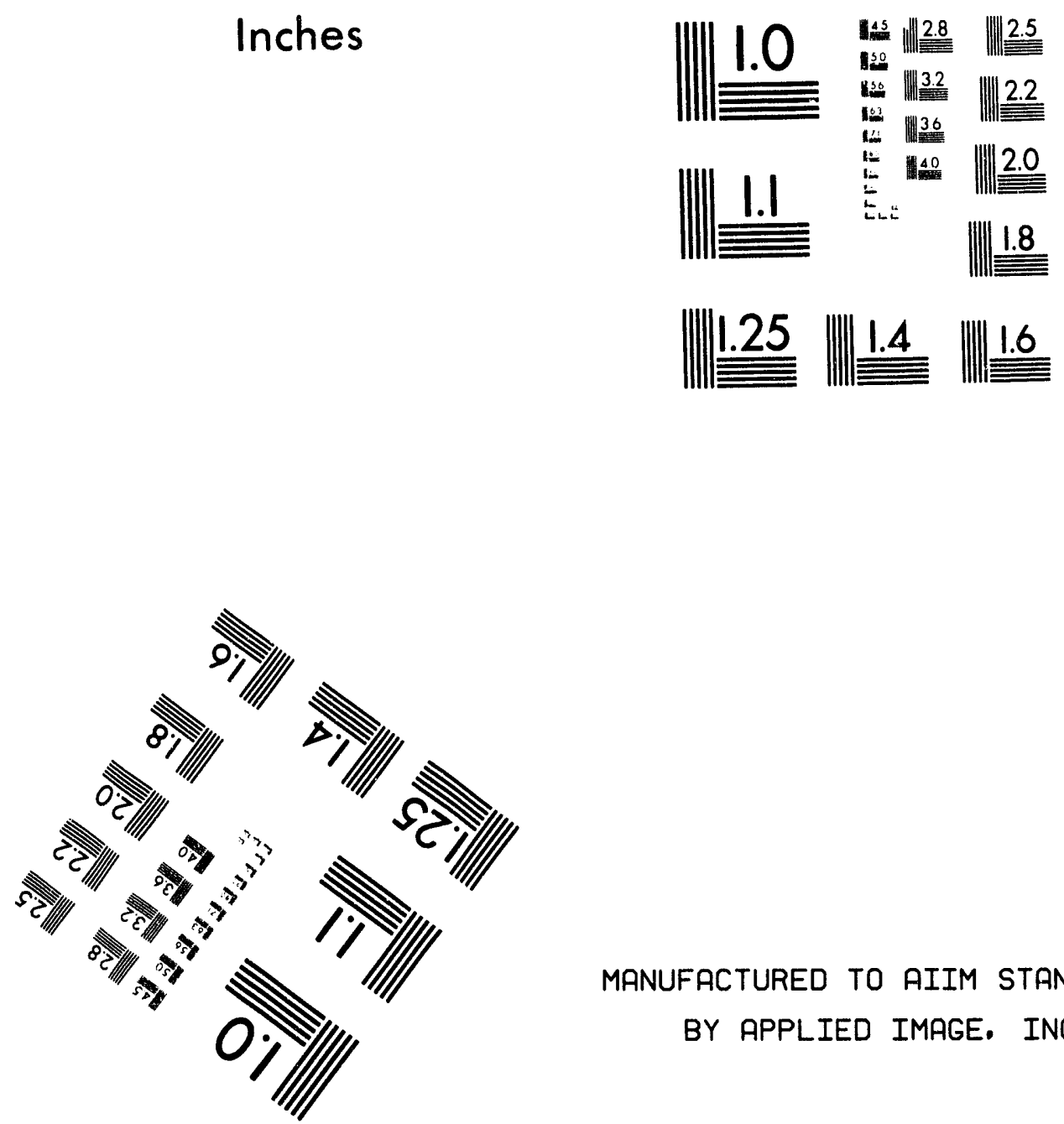

MANUFACTURED TO AIIM STANDARDS

BY APPLIED IMAGE. INC.

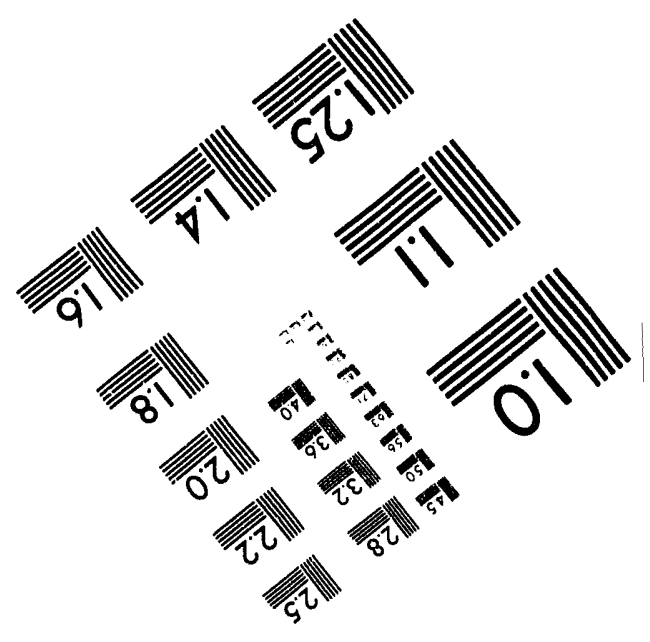



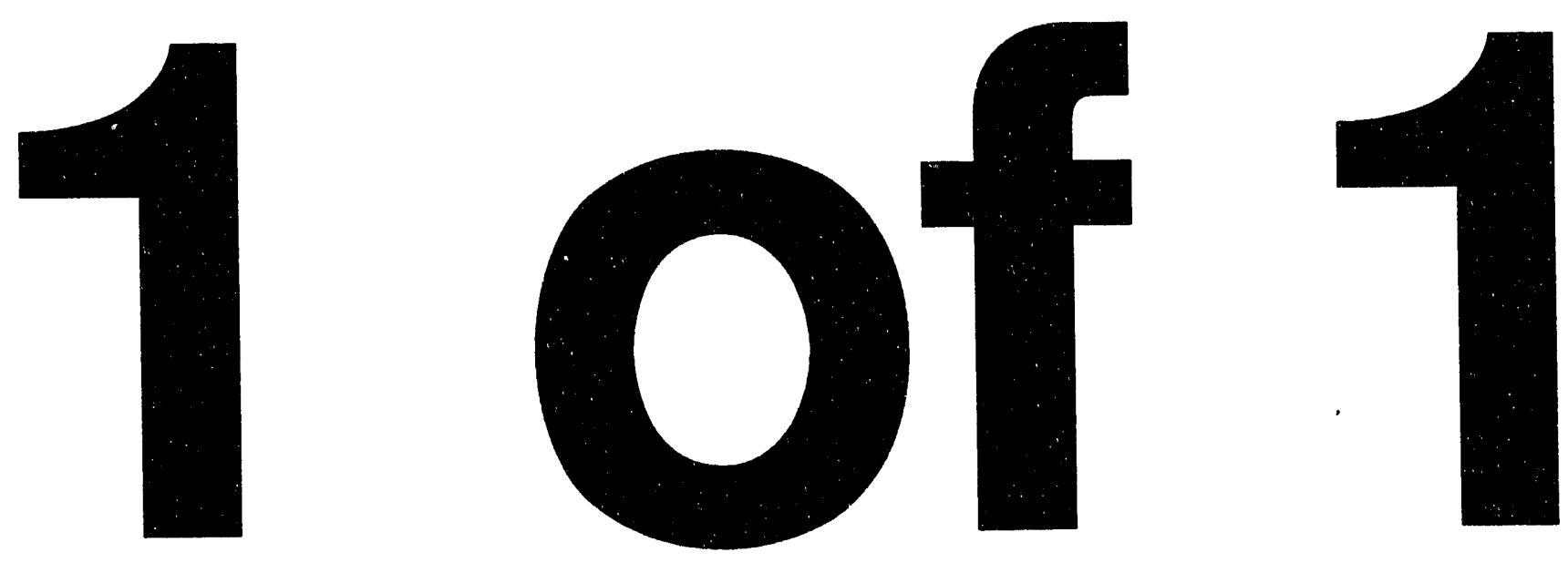


\title{
CHARACTERIZATION OH. NSLS ACCELERATING CAVITIES USING IMPEDANCE MEASUREMENT TECHNIQUES
}

\author{
S.M. Hanna and P.M. Stefan \\ National Synchrotron Light Source \\ Brookhaven National Laboratory \\ Upton, NY 11973
}

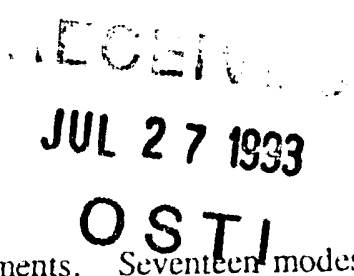

\section{Abstract}

Impedance measurements, using a central wire to simulate the electron beam, were performed on a $52 \mathrm{MHz}$ accelcrating cavity at the National Synchrotron Light Source (NSLS). To damp higher-order modes (HOM) in this cavity, damping antennas have been installed. We implemented the impedance measurement technique to characterize the cavity modes up to $1 \mathrm{GHz}$ and confirm the effectiveness of the damping antennas. Scattering parameters were measured using a network analyzer (HP 8510B) and values for $R$ and $Q$ were extracted using a new analytical technique. Our results showed good agreement with URMEL simulations and with other independent $Q$ measurements. This technique offers a more time-effective technique for obtaining $R / Q$, compared with the bead-pull method.

\section{HOM MEASUREMENTS ON THE NSLS 52 MHZ CAVITY}

As part of an upgrade for the X-ray Storage Ring at the NSLS, a fourth $52 \mathrm{MHz}$ cavity was recently installed. To damp higher-order modes (HOM) in this cavity, five damping antennas have been installed, as shown in Fig 1 . To characterize the cavity modes and the effectiveness of this damping technique, we implemented the impedance measurement technique [1-3], where a wire is introduced at the center of the component under test (the cavity). An if current is fed into this coaxial configuration and the resulting surface current distribution on the inner surface of the structure simulates the current distribution produced by a beam of charges. The existence of any discontinuity in the structure perturbs the current.

A central wire of radius $r_{1}=1.6 \mathrm{~mm}$ was fitted through the center of the cavity. Two spacers of $30 \mathrm{~cm}$ length and a radius $r_{2}=36.4 \mathrm{~mm}$ were connected to the cavity flanges, as shown in Fig. 2.a. The characteristic impedance of the spacers and the beam pipe in the cavity was $Z_{2}=187.5 \Omega$. Each spacer was inserted between a cavity flange and a coaxial cable $\left(Z_{1}=50 \Omega\right)$, which was connected to a port of the network analyzer. A frequency range from $45 \mathrm{MHz}$ to $1 \mathrm{GHz}$ was examined to locate higher-order modes. Any mode which produced a change in $S_{21} \geq 5 \mathrm{~dB}$ was included in the

\footnotetext{
Work performed under the auspices of the U.S. Department
} of Inereg. under contract DE- $\mathrm{ACO} 2-6(\mathrm{HO})(0) 16$. subsequent high-resolution measurements. Seventeen modes were identified with the damping antennas in place, and thirty with the antennas removed. Following these measurements, the gap in the cavity was shorted by inserting an expanding sheet-metal sleeve, and all forty-seven high-resolution scans repeated.

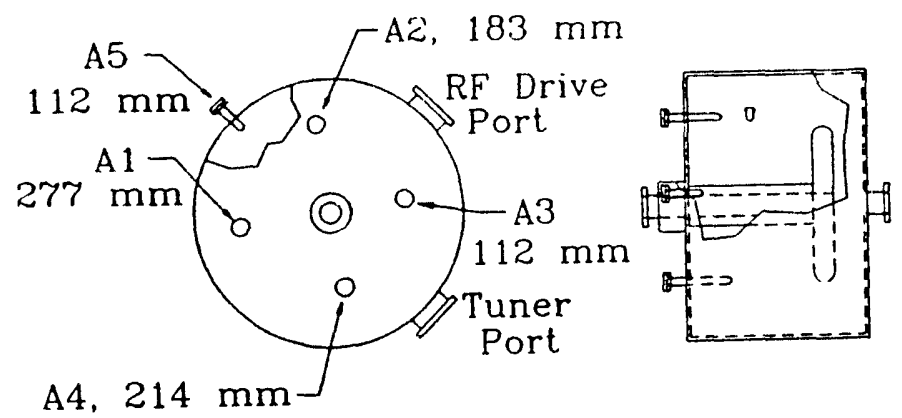

Fig. 1 HOM damping antennas in the $52 \mathrm{MHz}$ cavity.

\section{EXTRACTION OF CAVITY IMPEDANCES FROM SCATTERING PARAMETERS MEASUREMENTS}

Characterizing different modes of the $52 \mathrm{MHz}$ cavity involved evaluating the shunt resistance $R_{\text {sh }}$, and the quality factor $Q$, for each mode, before and after damping. This required calculating the impedance $Z(\omega)$ for each mode from the measured transmission response $S_{21}(\omega)$ for the system shown in Fig 2.a, and modeled in Fig 2.b.

The system was modeled as cascaded networks, each of which is described by an S-matrix. It is more convenient to describe each section of the system by a transfer matrix relating incident and reflected waves at one port to the incident and reflected waves at the other port [4]:

$$
\left(\begin{array}{l}
a_{2} \\
b_{1}
\end{array}\right)=\left(\begin{array}{ll}
T_{21} & T_{12} \\
T_{22} & T_{22}
\end{array}\right)\left(\begin{array}{l}
b_{2} \\
a_{2}
\end{array}\right)
$$

This form of network description is mathematically convenient for obtaining the overall T-matrix of the cascaded system. Since the network analyzer measurements are in terms of $S$ parameters, one needs to conven from the $T$-matrix to the $S$ matrix, using Eq. 2 .

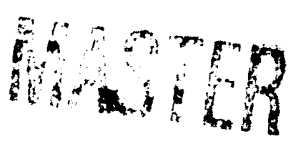




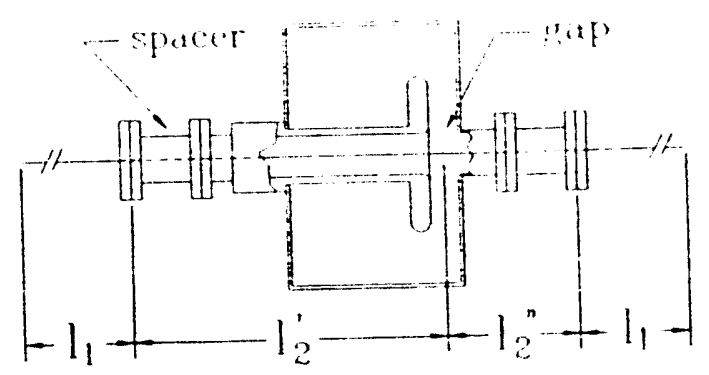

(a) System Configuration

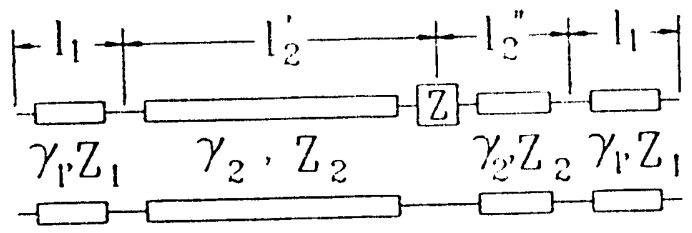

(b) Network Model

Fig. 2 Configuration for impedance measurement and the corresponding network model

$$
\left(\begin{array}{ll}
S_{11} & S_{12} \\
S_{21} & S_{22}
\end{array}\right)=\left(\begin{array}{cc}
\frac{T_{21}}{T_{11}} & T_{22}-\frac{T_{21} T_{12}}{T_{11}} \\
\frac{1}{T_{11}} & -\frac{T_{12}}{T_{11}}
\end{array}\right)
$$

Based on the network model shown in Fig 2.b, the $T$ parameters representation for the system can be written as:

$$
\begin{aligned}
& T=\left(\begin{array}{cc}
e^{\gamma_{1} l_{1}} & 0 \\
0 & e^{-\gamma_{1} l_{1}}
\end{array}\right)\left(\begin{array}{cc}
\frac{1}{1-\Gamma} & \frac{\Gamma}{1-\Gamma} \\
\frac{\Gamma}{1-\Gamma} & \frac{1}{1-\Gamma}
\end{array}\right)\left(\begin{array}{cc}
e^{\gamma_{2} l_{2}^{\prime}} & 0 \\
0 & e^{-\gamma_{2} l_{2}^{\prime}}
\end{array}\right) \\
& *\left(\begin{array}{cc}
\frac{Z+2 Z_{2}}{2 Z_{2}} & -\frac{Z}{2 Z_{2}} \\
\frac{Z}{2 Z_{2}} & \frac{2 Z_{2}-Z}{2 Z_{2}}
\end{array}\right) \\
& *\left(\begin{array}{cc}
e^{\gamma_{2} l_{2}^{\prime \prime}} & 0 \\
0 & e^{-\gamma_{2} 1_{2}^{\prime \prime}}
\end{array}\right)\left(\begin{array}{cc}
\frac{1}{1+\Gamma} & -\frac{\Gamma}{1+\Gamma} \\
-\frac{\Gamma}{1+\Gamma} & \frac{1}{1+\Gamma}
\end{array}\right)\left(\begin{array}{cc}
e^{r_{1} l_{1}} & 0 \\
0 & e^{-r_{1} l_{1}}
\end{array}\right)
\end{aligned}
$$

where,

$\gamma \equiv$ Propagation constant in each transmission line section. $\Gamma \equiv$ Reflection coefficient.

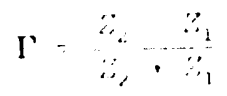

Conversion to S-parameters.

$$
\begin{aligned}
& S_{21}=\left(1-\Gamma^{2}\right) /\left(\frac{Z+2 z_{2}}{2 z_{2}}, \cdots \cdots+\cdots \cdot u^{\prime \prime}\right. \\
& +\left(\frac{Z}{2 Z_{2}}\right) \Gamma e^{2-y_{1} \cdot C} \cdot \therefore
\end{aligned}
$$

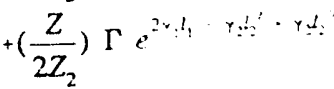

$$
\begin{aligned}
& \left.-\left(\frac{2 Z_{2}-Z}{2 Z_{2}}\right) \Gamma 2 e^{2 I_{1} d_{1}-(1,2 \cdot 1: 2)}\right]
\end{aligned}
$$

Solving for the cavity impedance gives

$$
Z=\frac{\left[\frac{2 Z_{2}}{S_{21}}\left(1-\Gamma^{2}\right) e^{-2 \gamma_{1} l_{1}}-2 Z_{2} e^{\gamma L_{2}}\left(1-\Gamma^{2} e^{-2 \gamma \gamma_{\gamma}}\right)\right]}{\left[e^{\gamma_{2} h^{2}}\left(1+\Gamma^{2} e^{-2 \gamma_{2} h_{h}}\right)+\Gamma e^{\gamma_{2} \Delta h_{h}}\left(1+e^{-2 \gamma_{2} \Delta h_{h}}\right)\right]}
$$

where,

$$
l_{2}=l_{2}^{\prime}+l_{2}^{\prime \prime}, \quad \Delta l_{2}=l_{2}^{\prime}-l_{2}^{\prime \prime}
$$

A program was written to obtain the impedance $Z(\omega)$ (magnitude and phase) for each of the cavity modes from the measured S-parameter data. A normalization step was included to account for phase shift and attenuation in the network analyzer. The program then calculates $Z(\omega)$ according to Eq.(6) using corrected $S_{21}(\omega)$ values (in phase and magnitude). The resulting $\phi_{z}(\omega)$ and $|Z(\omega)|$ for each cavity mode serve as inputs to a search program to find shunt the resistance $R_{s h}$, the resonant frequency. $f$, and the $3 \mathrm{~dB}$-bandwidth. As a sample of the results obtained, the extracted $Z(\omega)$ for the cavity HOM at $\mathrm{f}=275.1 \mathrm{MHz}$, with and without damping antennas installed, is shown in Fig 3.
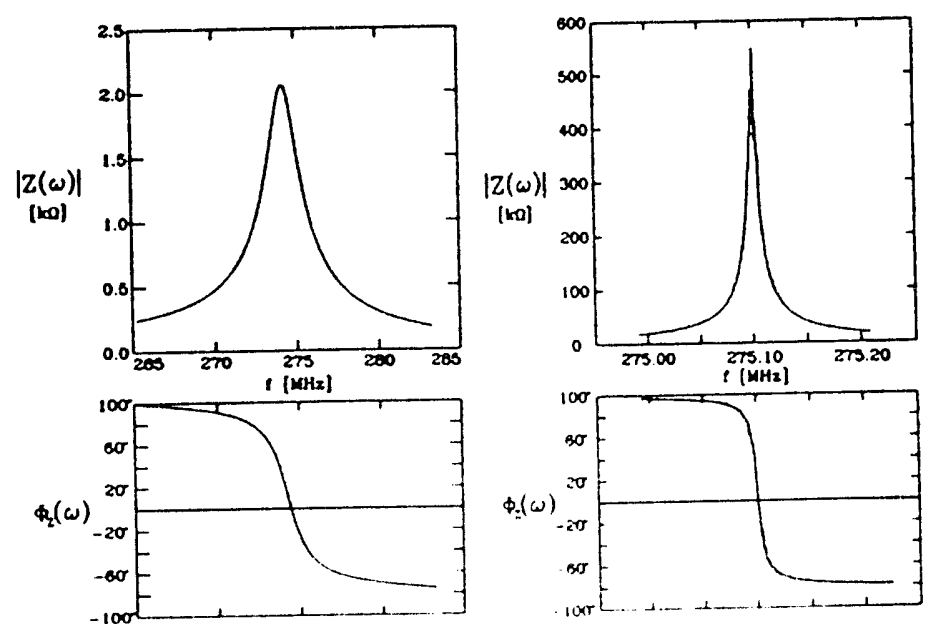

Fig. 3 Mode $(\mathrm{f}=275.1 \mathrm{MHz})$ impe dance with and without damping antenras installed. 
TABIE I - RESULTS AND COMPARISONS

\begin{tabular}{|c|c|c|c|c|c|c|}
\hline \multirow{4}{*}{$\mathrm{f}\left(\mathrm{MH} \mathrm{H}_{2}\right)$} & \multirow{2}{*}{\multicolumn{2}{|c|}{ 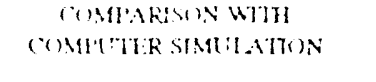 }} & \multicolumn{4}{|c|}{ 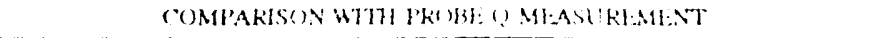 } \\
\hline & & & \multicolumn{2}{|c|}{ UNDAMPIED } & \multicolumn{2}{|c|}{ DAMIIID } \\
\hline & \multicolumn{2}{|c|}{$\mathrm{R} / \mathrm{Q}(\mathrm{B})$} & \multicolumn{2}{|c|}{$Q$} & \multicolumn{2}{|c|}{3.2} \\
\hline & THIS WOSK & URMAL & THIS WORK & PROHES & THIS WORK & PROBES \\
\hline 53.2 & 66.053 & 64.102 & 10,471 & $16.1(10)$ & 7598 & 13800 \\
\hline 275.1 & 14.156 & 14.848 & 36.122 & 33.050 & 145.0 & 160 \\
\hline 338.1 & 0.413 & 0.209 & 26.663 & 26,500 & $\cdots$ & 30 \\
\hline 397.8 & 5.492 & 5.240 & 27.367 & 26.800 & - & 30 \\
\hline 511.2 & 6.302 & 18.034 & 23,342 & 26.450 & $\cdots$ & 200 \\
\hline 586.1 & 20.555 & 15.129 & 29.447 & $\cdots$ & 333.7 & - \\
\hline 663.6 & 0.323 & 0.213 & 37,092 & 37,500 & 1697 & 1900 \\
\hline 713.8 & 0.260 & 0.347 & 44,822 & 44,000 & - & 180 \\
\hline 756.8 & 0.066 & 0.045 & 35,746 & 37,100 & 3355 & 3600 \\
\hline 792.3 & 2.556 & 3.882 & 14.806 & 10,750 & 1737 & 1900 \\
\hline 878.2 & 29.799 & 13.869 & 22,260 & 22,680 & 817.7 & 420 \\
\hline 954.7 & 0.007 & 0.211 & 30,329 & 48,900 & 2729 & 200 \\
\hline 975.4 & 1.519 & 1.635 & 6,471 & 6.080 & 1493 & 340 \\
\hline
\end{tabular}

\section{RESULTS AND COMPAISON WITH CAVITY SIMULATIONS AND WITH PROBE Q-MEASUREMENTS}

A partial summary of our results, together with various comparisons, is presented in Table 1. An important quantity to characterize a cavity mode is its geometrical impedance $\mathrm{R} / \mathrm{Q}$. We compared our measured values of $R / Q$ to values obtained from URMEL computer simulations. Examining the field patterns for different cavity modes using URMEL simulations reveals some explanation for the variation in agreement between measured and calculated values of $R / Q$. We noticed that when the fields associated with a certain mode are more concentrated in the volume close to the gap, the agreement is generally not as good, compared to other modes. Based on this observation, we argue intuitively that the perturbation introduced by the wire in the measurement has more effect on the field in such modes.

Our measured values for the $\mathrm{Q}$ were compared with other independent $Q$ measurements, where transmitting and receiving probes were placed on axis [5]. These resuits are also listed in Table 1.

\section{CONCLUSION}

The impedance meastrement technique was applied to the characterization of higher-onder modes in a $52 \mathrm{MHz}$ accelerating cavity at the NSLS. The results of the measurements are in good agreement with computer simulations for this cavity and in reasonable agreement with other independent measurement methods. We have developed and implemented a technique to extract the impedances of different modes from the measured S-parameters. Our analytical technique is a convenient alternative to the two approaches commonly used, namely, tapered transitions or a full calibration, which are normally needed in this type of measurement.

\section{References}

[1] F. Caspers, Frontiers of Particle Beams: Intensity Limitation, Springer-Verlag, pp. 80-109, 1992.

[2] L.S. Walling et al., "Transmission-Line Impedance Measurements for an Advanced Hadron Facility" Nucl. Instru. and Meth. in Phys. Res., A281, pp. 433-437, 1989.

[3] S.M. Hanna and P. Stefan, "Application of Impedance Measurement Techniques to Accelerating Cavity Mode Characterization", submitted to Nucl. Instru. and Meth. in Phys. Res.

14] R.E. Collin, Ficld Theory of Guided Waves, New York, McGraw Hill, Ch. 3, pp.79-83, 1960.

15] R. Biscardi and W. Bromerne, Private communication. 

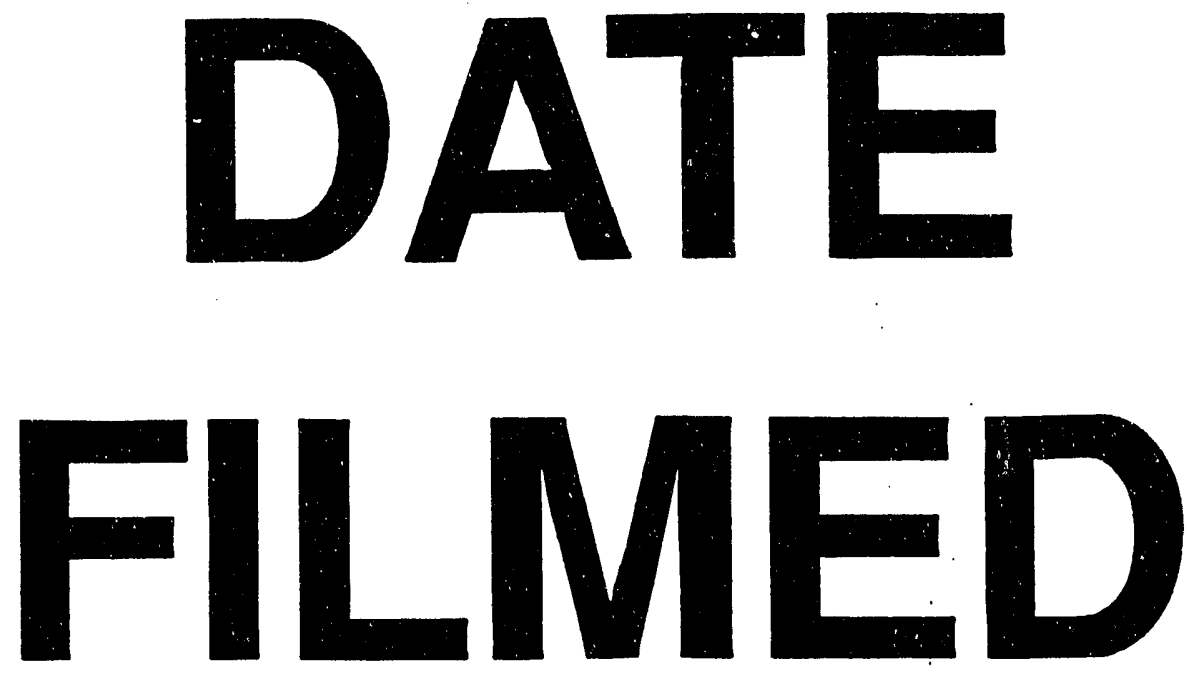

$9 / 9 / 93$
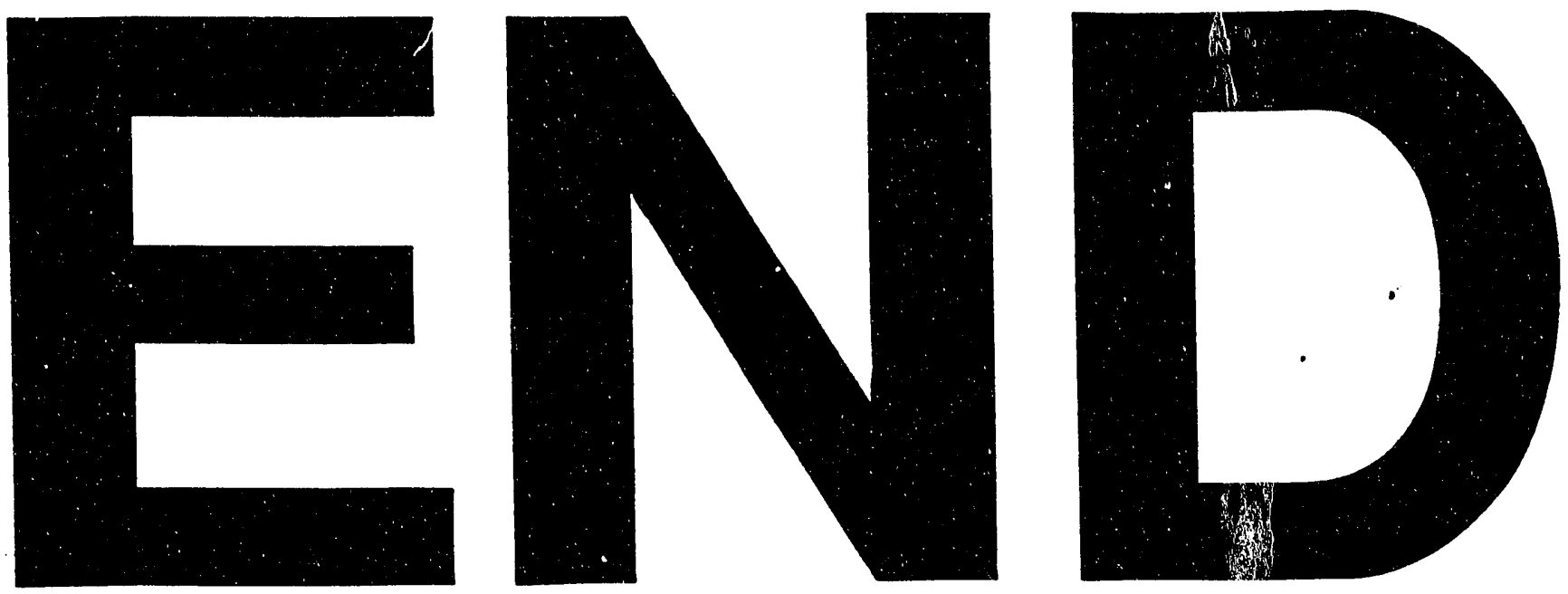
